\title{
Ultrahigh-Speed Dynamics of Micrometer-Scale Inertial Cavitation from Nanoparticles
}

\author{
J. J. Kwan, ${ }^{1}$ G. Lajoinie, ${ }^{2}$ N. de Jong, ${ }^{3}$ E. Stride,,${ }^{1}$ M. Versluis,,${ }^{2}$ and C. C. Coussios ${ }^{1, *}$ \\ ${ }^{1}$ Institute of Biomedical Engineering, University of Oxford, Oxford OX3 7DQ, United Kingdom \\ ${ }^{2}$ Physics of Fluids Group, MESA+ Institute for Nanotechnology, \\ MIRA Institute for Biomedical Technology and Technical Medicine, University of Twente, P.O. Box 217, \\ 7500 AE Enschede, The Netherlands \\ ${ }^{3}$ Biomedical Engineering, Erasmus Medical Center, P.O. Box 2040, 3000 CA Rotterdam, The Netherlands \\ (Received 13 March 2016; revised manuscript received 20 July 2016; published 3 October 2016)
}

Direct imaging of cavitation from solid nanoparticles has been a challenge due to the combined nanosized length and time scales involved. We report on high-speed microscopic imaging of inertial cavitation from gas trapped on nanoparticles with a tunable hemispherical depression (nanocups) at nanosecond time scales. The high-speed recordings establish that nanocups facilitate bubble growth followed by inertial collapse. Nanoparticle size, acoustic pressure amplitude, and frequency influence bubble dynamics and are compared to model predictions. Understanding these cavitation dynamics is critical for applications enhanced by acoustic cavitation.

DOI: 10.1103/PhysRevApplied.6.044004

\section{INTRODUCTION}

Acoustic cavitation is the nucleation and subsequent dynamics of a bubble due to an acoustic pressure wave. It is a key mechanical trigger for biomedical therapy and diagnosis (e.g., enhanced drug transport and passive acoustic mapping) [1,2] and industrial applications, such as sonochemistry $[3,4]$ and surface cleaning $[5,6]$. In the specific context of cancer therapy, acoustic cavitation has been shown in animal and more recently human studies to be critical in enhancing the delivery of a therapeutic agent from blood vessels and into tumors [1].

Though acoustic cavitation is crucial for the aforementioned applications, detrimental off-target effects may occur due to the large pressure amplitudes required to initiate cavitation in biological media [7]. For excitation frequencies in the range $0.5-3 \mathrm{MHz}$ typically used in biomedical ultrasound, previous work has shown that micrometer cavitation nuclei, e.g., microbubbles and solid particles, reduce the needed pressure amplitudes to below $2 \mathrm{MPa}$ [8-10].

However, several applications are not adequately addressed by micrometer-sized cavitation nuclei, especially those where a physical pore size or spatial feature prevents the delivery of microbubbles to lower the local cavitation threshold. Such applications may include dental cleaning [11], biofilm removal, and microbial decontamination of liquids and surfaces. For biomedical applications, cavitation-enhanced drug transport into tumors necessitates a cavitation agent that can cross from the bloodstream into the tumor mass through endothelial gaps that range in size between 100-800 nm, delivering sustained cavitation both in the bloodstream and in the tumor tissue.

*constantin.coussios@eng.ox.ac.uk
Previously, we reported on a stable and biocompatible formulation of size-controlled submicrometer nuclei that demonstrated prolonged circulation and exclusively produced inertial cavitation upon ultrasound exposure [12]. These submicrometer particles have a definitive "cup" shape (henceforth referred to as nanocups), which traps gaseous nucleation sites that initiate cavitation. Furthermore, nanocups enabled the enhanced delivery of novel therapeutic agents in tumors, including therapeutic antibodies [12] and oncolytic viruses [13], achieving as much as a 2-orders-ofmagnitude increase in the dose of therapeutic agent delivered to tumors.

Though there has been substantial work on the cavitation behavior of microparticles [14,15], little is known about the behavior of bubbles nucleated from solid nanoparticles such as nanocups. The physics of inertial cavitation from nanobubbles stabilized on nanoparticles are expected to differ from those of microparticles for several reasons. First, the stability of such nanobubbles on surfaces and in cavities remains poorly understood despite the ample evidence that they remain stable in cavities [16-18]. Second, for an acoustically driven bubble, the acoustic pressure required to drive inertial cavitation is expected to increase with a decreasing bubble size owing to the increasing effect of surface tension [19]. Third, compared to the recently wellstudied cavitation of nanobubbles from nanopits on large solid surfaces [20], cavitation from nanobubbles on nanoparticles is expected to differ by virtue of the fact that the expanding bubble does not have an effectively infinite solid surface along which it can grow during rarefaction.

In order to begin to describe these phenomena, we developed and published [21] a modified Rayleigh-Plesset model that accounts for the size and geometry of the nanocup. In the experimental part of this same study [21], 
it had only been possible to confirm certain aspects of the model through indirect observations of the acoustic emissions produced by the nanoparticles. In particular, the theoretical model was found to provide an accurate prediction of the acoustic pressure amplitude thresholds for the onset of inertial cavitation as a function of nanobubble size and frequency. However, purely acoustic experimental methods were unable to confirm the radial dynamics, maximum expansion diameter, rebound characteristics, and other cavitation behaviors predicted by the model.

The aim of the present work is to provide direct optical observations of cavitation initiation and subsequent growth and collapse of cavitation bubbles from solid nanoparticles, to enable confirmation of our recently published model [21], as well as an improved understanding of the effect of frequency and cavity size on the instigation and subsequent evolution of cavitation activity from solid nanoparticles. Validation of the predicted bubble dynamics will enable optimization of the microstreaming, shear stresses, and microjetting produced from such particles, enhancing the safety and efficacy of these systems for a broad range of biomedical and nonbiomedical applications.

\section{MATERIALS AND METHODS}

\section{A. Nanoparticle synthesis}

We fabricate three differently sized nanocups, as previously described [12]. In short, a seeded polymerization technique is used to coat a template polystyrene nanosphere with a copolymer of methyl methacrylate and divinylbenzene. Each nanocup suspension is measured three times by dynamic light scattering (ZetaSizerNano, Malvern). The average and standard deviations of the cumulative average of each distribution for the three differently sized nanocups are $174 \pm 2,425 \pm 6$, and $609 \pm 8 \mathrm{~nm}$. The size distribution widths are 57, 121, and $164 \mathrm{~nm}$ (respectively), which indicates that the distributions are monodisperse and narrow.

We categorize these particles as small, medium, and large based on their diameter. Transmission electron microscope images (TEM; Hitachi H-7650) display a single predominant depression on the surface of the nanocups (Fig. 1). Unless otherwise specified, all nanocups are air dried using an optimized drying and resuspension method developed previously [12,21]. After resuspension, all particles are diluted a hundredfold before study, unless otherwise stated.

\section{B. Experimental setup}

Figure 1 also shows the setup used to observe cavitation bubbles nucleated from the nanocups. Nanocups from each size class are placed in turn into an Opticell® (Thermo Scientific, UK) with dimensions $70 \times 65 \times 3 \mathrm{~mm}$ in length, width, and depth (respectively) at concentrations between $10^{7}$ and $10^{9}$ particles per ml. A focused $1-\mathrm{MHz}$ ultrasound source (Sonic Concepts H107), calibrated with a needle hydrophone ( $0.2 \mathrm{~mm}$, Precision Acoustics, UK), are

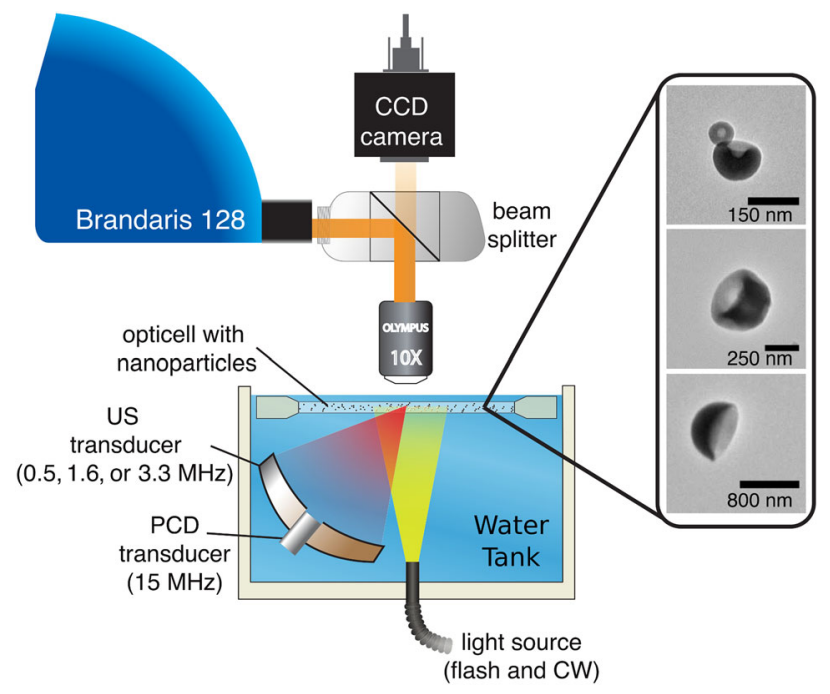

FIG. 1. Schematic of the experimental setup. The ultrasound (US) transducer is confocally aligned to both a passive cavitation detector (PCD) and the optical focus of the ultra-high-speed camera Brandaris 128. The nanoparticles are contained in an optically and acoustically transparent Opticell culture cell. The light source used was a cool white (CW) lamp. The three images to the right display transmission electron microscopy (TEM) images of small (174 $\pm 2 \mathrm{~nm})$, medium (425 $\pm 6 \mathrm{~nm})$, and large $(609 \pm 8 \mathrm{~nm})$ nanocups are shown.

coaligned with the optical focus of an ultrahigh-speed imaging system, the Brandaris 128 camera [22]. Unless otherwise stated, a $5 \times$ magnification objective (Olympus) is attached to the imaging system. The optical focus is placed roughly $1 \mathrm{~mm}$ from the top of the Opticell® wall facing the magnification objective, thereby minimizing wall effects that have been shown to dampen microbubble oscillations and modulate the scattered pressure at distances below $0.5 \mathrm{~mm}$ from a boundary [23,24]. The camera is the master trigger that initiates an ultrasound pulse timed to the light source such that the acoustic wave hits the sample at the time the camera starts recording. For each recording, a series of six 128-frame recordings are obtained at frame rates of $10 \times 10^{6}$ frames per second. The first recording in each sequence is a no-ultrasound control, and the following five are insonified with a 20-cycle sine wave [25]. The driving frequencies tested are 0.5, 1.6, and 3.3 MHz.

Acoustic emissions generated by the cavitation bubbles are recorded with a passive cavitation detector $(\mathrm{PCD}$; V319-SU, $15 \mathrm{MHz}$ PZT, Panametrics, focal distance $74.5 \mathrm{~mm}$ ) coaxially and confocally aligned with the transmitting transducer. In order to detect the broadband emission away from the fundamental ultrasound driving frequency, the signal is passed through a custom-built 7.5-MHz high-pass filter, amplified 5 times prior to being stored on a digital storage oscilloscope (DPO3034, Tektronix). The collected data are postprocessed to obtain power spectral density curves of the received voltage trace (Fig. S1, Ref. [25]) to confirm the presence of inertial 
cavitation $[12,21]$. In order to ensure that the nanocups are the sole cause of cavitation nucleation, 200-nm-filtered pure deionized water is exposed to two extreme acoustic parameters $(0.5 \mathrm{MHz}$ at $3.5 \mathrm{MPa}$ and $3.3 \mathrm{MHz}$ at $10 \mathrm{MPa})$. Neither of these extremes display broadband power exceeding $2 \mathrm{~dB}$ above the noise level, as opposed to over $10 \mathrm{~dB}$ when cavitation is observed. Similarly, nondried medium nanocups do not initiate inertial cavitation.

\section{RESULTS AND DISCUSSION}

Figure 2 displays representative images of temporal slices from ultrahigh-speed recordings of nanocups of different sizes at a $0.5-\mathrm{MHz}$ driving frequency and of medium nanocups at $1.6-$ and $3.3-\mathrm{MHz}$ driving frequencies. The peak rarefactional pressures used in this figure at each frequency always exceed the inertial cavitation threshold of the nanocups, namely $1.5 \mathrm{MPa}$ at $0.5 \mathrm{MHz}, 2.5 \mathrm{MPa}$ at $1.6 \mathrm{MHz}$, and $3.5 \mathrm{MPa}$ at $3.3 \mathrm{MHz}$. It is important to note that the nanocups themselves cannot be resolved optically and that only imaging of the bubble nucleated from the nanocup upon ultrasound exposure is possible.

Despite the asymmetry of the nanocup structure, it is evident that the initial expansion of the bubble is always spherical, for all frequencies and nanocup sizes, with the duration of bubble growth being roughly equal to the rarefactional half-period in all cases. This expansion also invariably results in a peak diameter that exceeds the initial

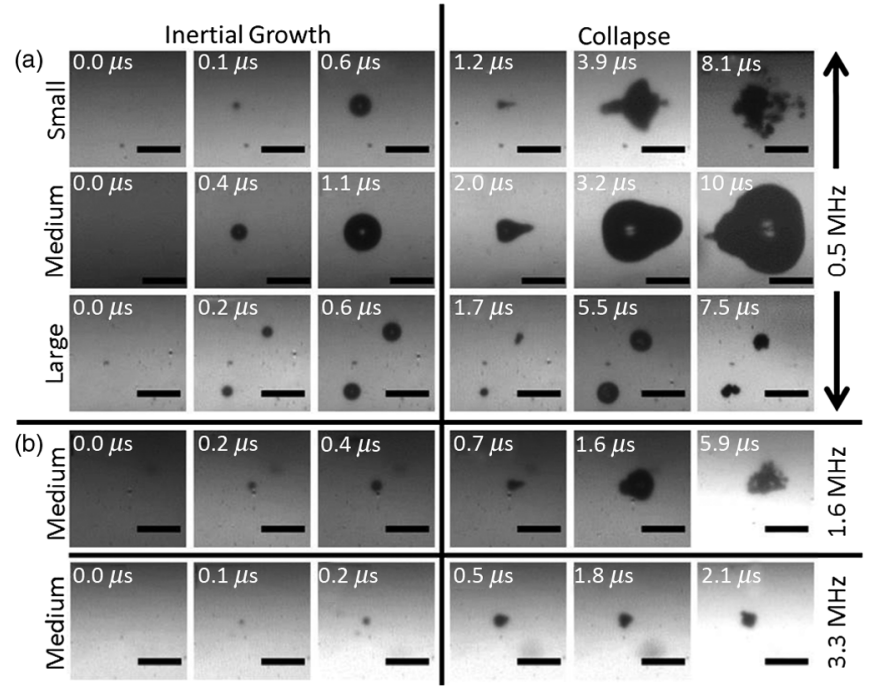

FIG. 2. Single images from the ultrahigh-speed recordings. (a) These snapshots show the response of nanocups exposed to ultrasound driven at $0.5 \mathrm{MHz}$ and $3.5 \mathrm{MPa}$ as a function of size. (b) Bubble behavior at constant size exposed to a $1.6-$ or $3.3-\mathrm{MHz}$ driving frequency are also shown. The driving pressure amplitudes are 6.0 and $7.0 \mathrm{MPa}$, respectively. Across all test conditions, the recordings are dividable into two regimes, the inertial growth phase and the bubble collapse phase. The initial time point $(0.0 \mu \mathrm{s})$ is the frame before the first bubble is observed. The scale bars in all images are $100 \mu \mathrm{m}$. bubble radius by 1 to 2 orders of magnitude, crossing the Blake threshold [26] and suggesting a cavity that predominantly consists of water vapor. However, as summarized in Figs. 4(c)-4(e), this maximum diameter only appears to depend on frequency, but is surprisingly independent of the initial nanocup and bubble diameter.

Shortly after maximum expansion, Fig. 2 shows that the bubble inertially collapses during the compressional phase of the ultrasound wave. In all cases, this collapse is highly asymmetric across all tested conditions, with its timing and violence once again unaffected by the initial nanocup or bubble size, driving frequency, or acoustic pressure amplitude. A broadband acoustic emission accompanies the violent collapse of the bubble in all observed instances of cavitation. Our observations, both optical and acoustic, confirm our previous assertion that nanocups either exhibit no response at all (below the cavitation threshold), or exclusively yield inertial cavitation (above the cavitation threshold) upon exposure to ultrasound.

We hypothesize that the inertial cavitation of bubbles nucleated from nanocups occurs in several stages [Fig. 3(a)]. Starting within the cavity of the nanocup, a nanobubble exposed to ultrasound will extend out of the cavity and detach as the contact angle of the growing bubble reaches a critical angle. Optical observations of this growth and subsequent detachment of a nanobubble from within a solid nanoparticle have not been previously reported and are extremely challenging, not least because the nanoparticle itself is just smaller than the optical resolution, and because capturing both a nanoparticle and the direction of bubble
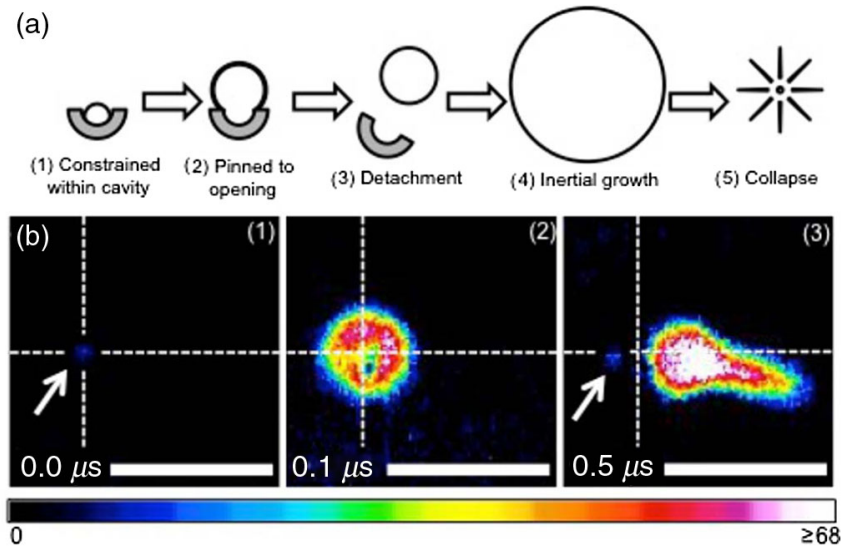

FIG. 3. Comparison of hypothesized mechanism and experimental observation. (a) An illustration of a surface-trapped nanobubble undergoing inertial collapse. (b) Evidence for a (1) nanocup likely with a trapped nanobubble, (2) nucleation, and (3) detachment from a single large nanocup is shown. Dashed lines indicate the initial position of the nanocup. Scale bars represent $100 \mu \mathrm{m}$. The arrow points to the 600-nm-diameter nanocup. The time stamps on the images are based on the onset of cavitation. The color bar is the difference in pixel intensity (of arbitrary units) in each frame of the video compared to the same frame number of the no-ultrasound control video. 


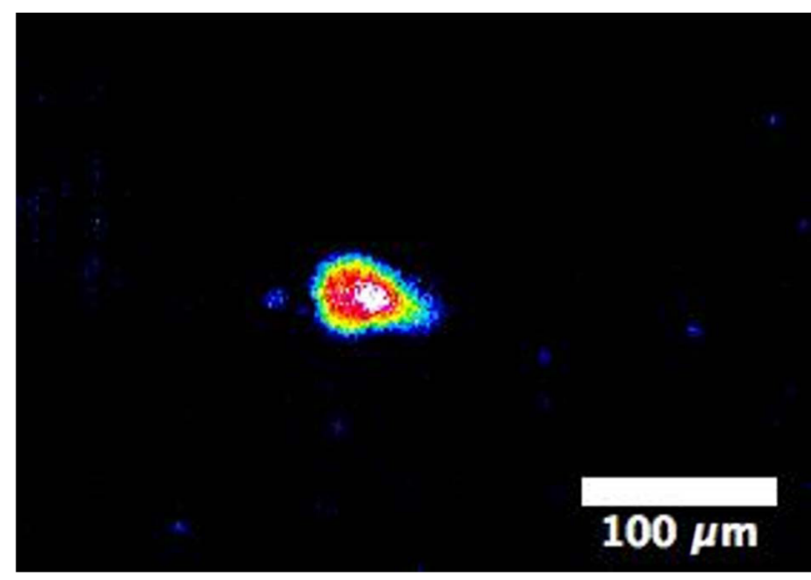

VIDEO 1. In this video, the scattering from a large nanocup is shown to nucleate a microbubble on the order of $100 \mu \mathrm{m}$. This video is background subtracted with the brightness and contrast adjusted. It is colorized and cropped to emphasize the location of the nanocup. The large nanocup is exposed to a 20-cycle sine burst at $6-\mathrm{MPa}$ peak negative-pressure amplitude at $1.6-\mathrm{MHz}$ driving frequency. The video is recorded with a $20 \times$ objective attached to the microscope at $10 \times 10^{6}$ frames per second and is being played back at 25 frames per second.

detachment in the same focal plane would require a serendipitous capture across thousands of recorded events.

Using our largest nanocups $(\sim 600 \mathrm{~nm})$ that are able to scatter enough light to be detectable by the camera, we are able to capture such a rare event in Video 1. In order to separate visual artifacts caused by the camera and videoprocessing algorithm from nanocups in view, we subtract each video frame with the corresponding no-ultrasound control video frame. Next, we adjust for brightness and contrast, and later colorize the recordings to emphasize their location. Images from this video are taken at three different time points [Fig. 3(b)] and appear to show a single large nanocup nucleating a bubble that detaches and jets away from a large nanocup, providing initial evidence in support of our hypothesis for bubble growth and detachment. The nanocup is immediately engulfed by the bubble cloud post collapse. Though previous work [15,20,27] has reported similar phenomena at much larger length scales, this is preliminary evidence of controlled bubble detachment from a submicron particle.

To verify our previously described Rayleigh-Plesset crevice model [21], we evaluate the bubble dynamics, defined by $R \ddot{R}+(3 / 2) \dot{R}^{2}=\left(1 / \rho_{L}\right)\left[P_{L}(R)-P_{\mathrm{ac}}(t)-P_{0}\right]$, where $R, \dot{R}$, and $\ddot{R}$ are the radius, velocity, and acceleration of the bubble wall. The density of water is $\rho_{L}$, the liquid pressure at the bubble wall is $P_{L}$, the acoustic pressure amplitude is $P_{\text {ac }}$ at a given time $t$, and $P_{0}$ is the ambient pressure. As described in our crevice model [21], the radius of the bubble depends on whether the bubble lay within, is on the surface, or has detached from the nanocup cavity. We have previously shown that this model adequately describes the cavitation threshold from solid gas-stabilizing submicron particles [21], but had been unable to obtain direct validation of the predicted radiustime curves. In order to enable a comparison of the model predictions to our optical observations (Fig. 4), the only model parameter that requires adjustment is the initial cavity diameter. To achieve this, we iteratively chose an initial cavity diameter that falls within the range of measured cavity sizes for this particular nanocup batch (e.g., 200-400 nm for medium nanocups) to match the peak diameter of the model to that recorded optically at the particular excitation pressure and frequency used in the experiment. (a)
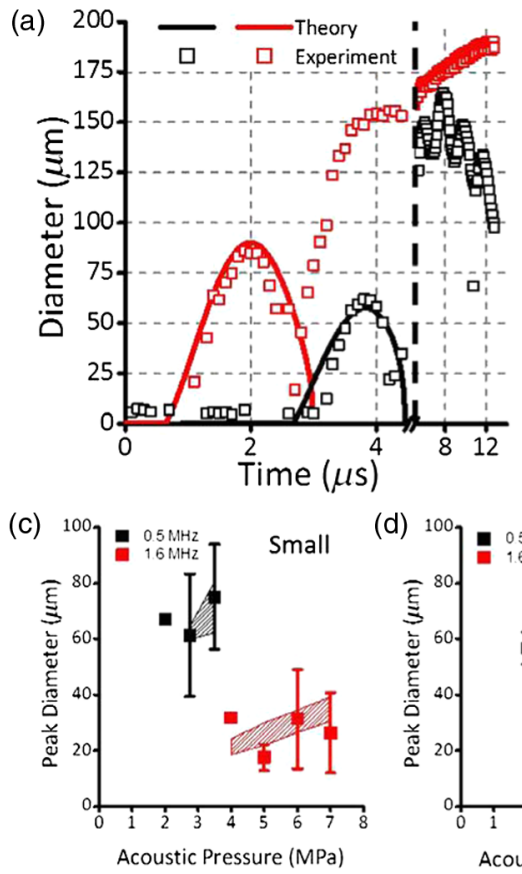

(d)

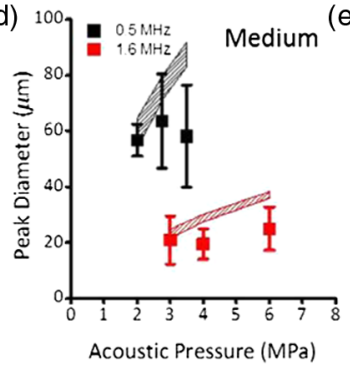

(b)

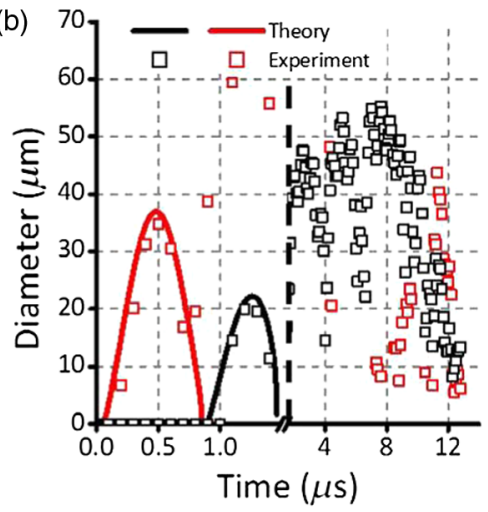

(e)

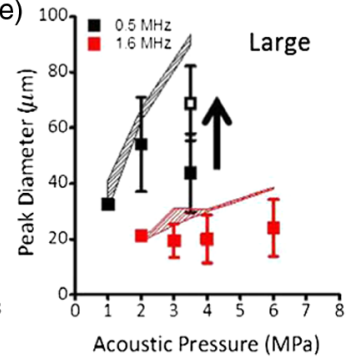

FIG. 4. A comparison between the predicted and observed cavitation behavior. (a),(b) A good agreement is found between the diameter-time curve predicted by the modified Rayleigh-Plesset crevice model and representative experimentally observed bubble diameter at (a) $0.5 \mathrm{MHz}$ [at $2 \mathrm{MPa}$ (black) and $3.5 \mathrm{MPa}$ (red)] and (b) $1.6 \mathrm{MHz}$ [at $3 \mathrm{MPa}$ (black) and $6 \mathrm{MPa}$ (red)] for medium-sized nanocups. The dashed line in the location of the time axis break marks the bubble transition from spherical collapse (left of the line) to nonspherical collapse (right of the line). (c)-(e) Peak diameters achieved during inertial expansion for (c) small, (d) medium, and (e) large nanocups at $0.5 \mathrm{MHz}$ (black) and 1.6 MHz (red) are shown. Shaded regions are predicted peak diameters. The single open square in (e) is from measurements taken at concentrations 10 times lower than those for the closed squares. Values without error bars are due to only a single bubble captured at that particular acoustic setting. 
This comparison (Fig. 4) yields good agreement between the modeled and experimentally observed radius-time curve at two different driving frequencies, $0.5 \mathrm{MHz}$ [Fig. 4(a)] and 1.6 MHz [Fig. 4(b)], and two different acoustic pressure amplitudes. The dynamics at $3.3 \mathrm{MHz}$ cannot be reliably extracted as the bubbles formed are at the resolution limit of the imaging system during this initial growth and first collapse (Fig. 2).

Very good agreement is also achieved between model and experiment in terms of the peak diameter reached by the nanobubbles at both 0.5 and $1.6 \mathrm{MHz}$ across a range of excitation pressures [Figs. 4(c)-4(e)]. As expected, the higher frequency results in consistently smaller peak diameters being reached for a given initial nanobubble size. However, somewhat surprisingly, rarefactional pressure amplitude has less of an effect across each nanocup size as compared to the theoretical predictions. In fact, the main consequence of increasing the peak rarefactional pressure amplitude is to increase the number of cavitation events per frame (Fig. 5) rather than the peak diameter of these events.

We hypothesize that this trend is due to increasing interactions between bubbles as the incidence of cavitation increases, and to acoustic shielding of the incident sound field from nearby and previously nucleated microbubbles that reduces the acoustic pressure experienced by the nucleating cup. In order to test this hypothesis, we further exposed large nanocups diluted by an order of magnitude $\left(10^{6}\right.$ particles per $\left.\mathrm{ml}\right)$ to ultrasound at 0.5 and $3.5 \mathrm{MPa}$.
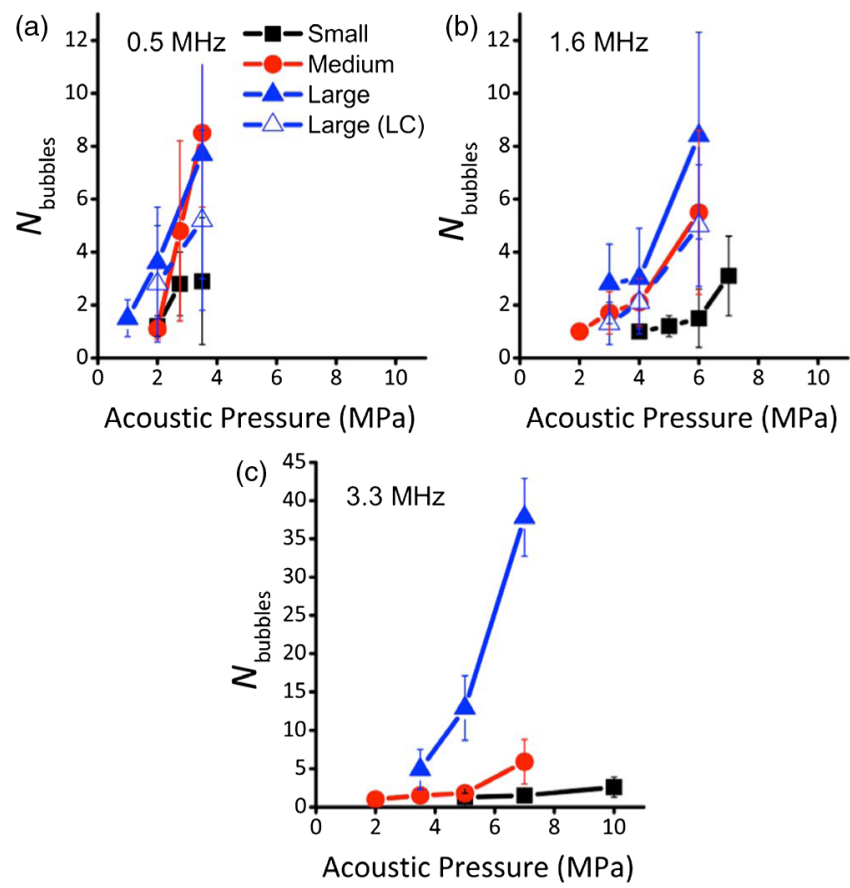

FIG. 5. Recorded number of bubbles per first recording ( $\left.N_{\text {bubbles }}\right)$ for small, medium, and large nanocups at (a) $0.5-\mathrm{MHz}$, (b) $1.6-\mathrm{MHz}$, and (c) $3.3-\mathrm{MHz}$ driving frequencies. The legend in (a) is valid for all figures, and LC represents the low concentration (tenfold dilution) of large nanocups.
At this lower concentration, the peak bubble diameter is in better agreement with that predicted by the model [Fig. 4(e), open square data point], confirming that the lower concentration corresponds more closely to the singlebubble scenario described by the model.

After the inertial growth, Fig. 2 shows that the nucleated bubbles undergo a chaotic and violent collapse phase. After the first collapse, the model is unable to predict the behavior of the bubbles due to asymmetry and breakup of the bubble, features that are not included in the model. We also observe that most bubbles remain after collapse because of the energy supplied by the acoustic wave. These bubbles rapidly disappear once the field is removed, as we do not observe that any bubbles carry over to the next video. This further suggests that although the initial nanobubble on the surface is composed of air, the observed bubbles are likely predominantly made of water vapor.

Jetting always occurs once for every bubble nucleated with a preferential direction aligned with the propagation direction of the ultrasound wave (Fig. 2) [28]. However, local heterogeneities such as other nucleated bubbles strongly affect jet direction. For example, two bubbles nucleated near each other result with jets striking each other [9] and coalesce (Video 2). It is also possible that the walls of the sample container influence jetting. Jet size is dependent on the driving frequency, with the largest jet lengths (up to $100 \mu \mathrm{m}$ ) at $0.5 \mathrm{MHz}$. The jets also translate the center of the nucleated bubble in the direction of the jet itself [27].

Post jet formation, the violent cavitation dynamics that occur are generally nonspherical. Some bubbles grow to a size larger than $200 \mu \mathrm{m}$ in diameter, an increase in volume of approximately 9 orders of magnitude, taking up nearly

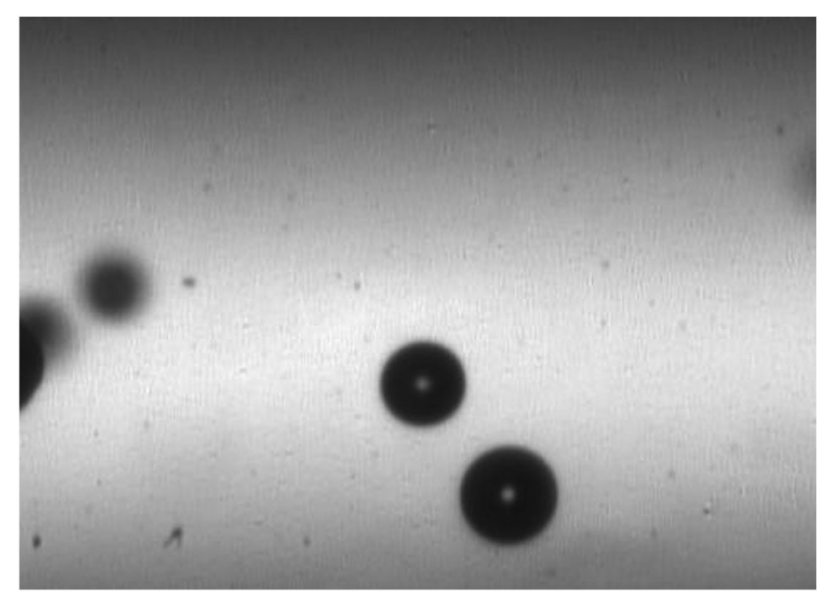

VIDEO 2. This video illustrates an example of two bubbles nucleated from large nanocups. Because of the proximity of the two bubbles, they jet towards each other and coalesce into a larger single bubble. The sample is exposed to a 20-cycle sine burst at 3.5 $\mathrm{MPa}$ peak negative pressure at $0.5 \mathrm{MHz}$ driving frequency. The video is recorded at $5 \times$ magnification and at $10 \times 10^{6}$ frames per second. It is played back at 25 frames per second. 


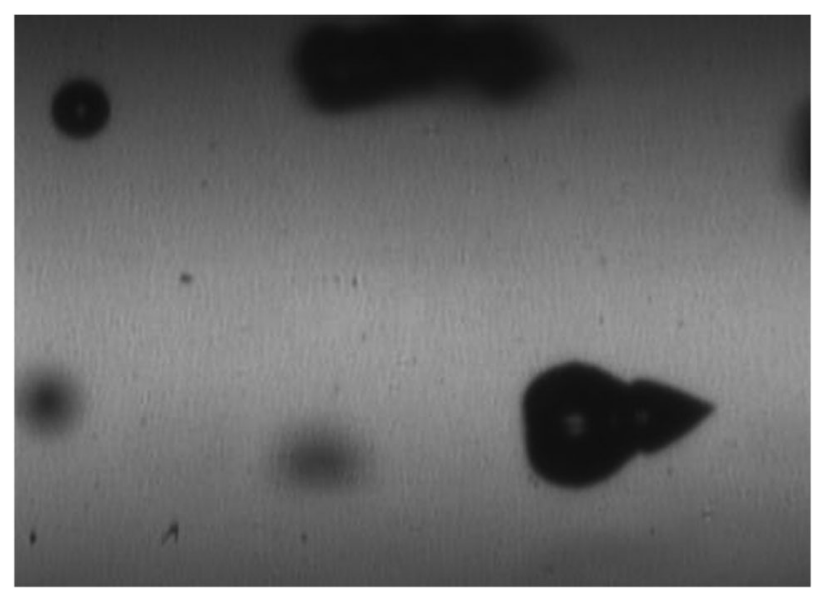

VIDEO 3. A bubble nucleated from a medium-sized nanocup is shown to jet and rebound through the center of the cavitation bubble. The jet involution is visible due to the size of the nucleated bubble. The sample is exposed to a 20 -cycle sine burst at $3.5 \mathrm{MPa}$ peak negative pressure at $0.5 \mathrm{MHz}$ driving frequency. The video is recorded at $5 \times$ magnification and at $10 \times 10^{6}$ frames per second. It is played back at 25 frames per second.

the entire field of view in the present setup. Such bubbles are large enough to observe the rebound of the jet passing through the center of the bubble (Video 3).

In our measurements, we observe that the first rebound is generally larger than the initial bubble expansion. We believe that this phenomenon may be due to several factors. First, the presence of air, a noncondensable gas, in the cavitating vapor bubble will hinder vapor condensation [29] and prevent complete collapse of the bubble during the compression phase of the ultrasound wave [30]. We also observe that the perceptible minimum bubble size during spherical collapse is larger [10 to $20 \mu \mathrm{m}$ at $0.5 \mathrm{MHz}$-Fig. 4(a)] than the initial bubble size. This now-larger vapor or gas bubble experiences the second rarefactional phase of the ultrasound wave, and grows to a bigger size compared to that of the initial spherical growth. Furthermore, the first collapse of the bubble results in a highly asymmetric jet, which suggests that there is increased energy for the rebound [31]. In addition, the resonant frequency of these asymmetric bubbles is also closer to the driving frequency. However, the exact reason for our observations post collapse remains unknown, and will be explored in future studies.

Bubbles also violently fragment into bubble clouds. These two modes of collapse dominate most of the lifespan of a nucleated bubble. This phenomenon, coupled with our previous observation that bubbles persist in the presence of the acoustic wave, suggests that these collapses occur throughout the entire acoustic wave, which is evidenced by cavitation persisting at larger pressure amplitudes (Fig. S2) [25]. These collapses likely govern macroscopic effects, such as microstreaming, that operate in the millisecond time scales. As a result, our observations on cavitation from solid nanoparticles may have a substantial impact on understanding the role of collapse mechanics on transport phenomena in size-critical biomedical and industrial applications, such as cavitation-enhanced cancer therapy [1].

Figure 5 shows the concentration of cavitation bubbles generated for each acoustic setting and sample from the first video in the series exposed to ultrasound. It is essential to note that the number of created bubbles is nowhere near the number of particles present in the medium. For the large particles, a further tenfold dilution only leads to under a twofold reduction of the number of observed cavitation events. This supports the idea that there are complex interactions between the bubbles and the acoustic field. The number of bubbles created significantly decreases with increasing frequency (at constant pressure). Increasing the frequency also increases the influence of the size of the nanoparticle: a threefold increase at $0.5 \mathrm{MHz}$ between small and large nanocups and a 33-fold increase at $3.3 \mathrm{MHz}$. Increasing the pressure had a similar effect on the number of cavitation bubbles with a nearly threefold increase when the pressure amplitude is increased from 5 to $7 \mathrm{MPa}$, and confers with previous results from surface nanopits [20].

\section{CONCLUSION}

Our understanding of the dynamics and behavior of cavitation bubbles from gas-stabilizing solid nanoparticles is critical for their use in both industrial and clinical applications. In this manuscript, we have successfully observed the dynamic and violent cavitation behavior of bubbles nucleated from nanocups of three different mean diameters at $10 \times 10^{6}$ frames-per-second frame rates using the Brandaris 128 ultrahigh-speed camera. Nanocups of all sizes nucleate inertial cavitation at all driving frequencies studied and only exclusively produce broadband emissions indicative of inertial cavitation. The nucleated bubble shows an initial inertial growth phase followed by detachment from the solid nanoparticle and subsequent spherical collapse, confirming the underpinning hypothesis and quantitative radius-time predictions of our modified Rayleigh-Plesset cavity model at two different frequencies. The peak inertial growth diameter is governed primarily by the ultrasound driving frequency, and is independent of the nanocup size or acoustic pressure. Ultrasound pressure amplitude, however, controls the number of bubbles nucleated. Nonspherical collapses are consistently observed, starting with a jet that is followed by continued growth and/or fragmentation within the observed time frame. These nanosecond events carry on for a majority of the bubble lifespan, suggesting that this behavior likely governs macroscopic time and length-scale phenomena through microstreaming and translation due to acoustic radiation forces. Bridging the gaps between the application and physics of this technology is critical in designing and utilizing these next-generation cavitation nuclei for both biomedical and industrial applications. 


\section{ACKNOWLEDGMENTS}

This work is supported by NanoNextNL, a micro- and nanotechnology consortium of the Government of The Netherlands and 130 partners, and by the U.K.'s Engineering and Physical Sciences Research Council under the OxCD3 Programme Grant (No. EP/L024012/1). We also warmly thank Gert-Wim Bruggert, Martin Bos, and Bas Benschop for their ongoing and effective technical support.

[1] R. Carlisle, J. Choi, M. Bazan-Peregrino, R. Laga, V. Subr, L. Kostka, K. Ulbrich, C. C. Coussios, and L. W. Seymour, Enhanced tumor uptake and penetration of virotherapy using polymer stealthing and focused ultrasound, J. Natl. Cancer Inst. 105, 1701 (2013).

[2] H. Chen, W. Kreider, A. A. Brayman, M. R. Bailey, and T. J. Matula, Blood Vessel Deformations on Microsecond Time Scales by Ultrasonic Cavitation, Phys. Rev. Lett. 106, 034301 (2011).

[3] D. Fernandez Rivas, P. Cintas, and H. J. G. E. Gardeniers, Merging microfluidics and sonochemistry: Towards greener and more efficient micro-sono-reactors, Chem. Commun. (Cambridge) 48, 10935 (2012).

[4] K. Yasuda, T. Torii, K. Yasui, Y. Iida, T. Tuziuti, M. Nakamura, and Y. Asakura, Enhancement of sonochemical reaction of terephthalate ion by superposition of ultrasonic fields of various frequencies, Ultrason. Sonochem. 14, 699 (2007).

[5] J. H. Bang and K. S. Suslick, Applications of Ultrasound to the Synthesis of Nanostructured Materials, Adv. Mater. 22, 1039 (2010).

[6] D. Fernandez Rivas, B. Verhaagen, J. R. T. Seddon, A. G. Zijlstra, L. M. Jiang, L. W. M. van der Sluis, M. Versluis, D. Lohse, and H. J. G. E. Gardeniers, Localized removal of layers of metal, polymer, or biomaterial by ultrasound cavitation bubbles, Biomicrofluidics 6, 034114 (2012).

[7] T. Li, H. Chen, T. Khokhlova, Y.-N. Wang, W. Kreider, X. He, and J.H. Hwang, Passive cavitation detection during pulsed HIFU exposures of ex vivo tissues and in vivo mouse pancreatic tumors, Ultrasound Med. Biol. 40, 1523 (2014).

[8] J. H. Hwang, J. Tu, A. A. Brayman, T. J. Matula, and L. A. Crum, Correlation between inertial cavitation dose and endothelial cell damage in vivo, Ultrasound Med. Biol. 32, 1611 (2006).

[9] J. J. Choi and C.-C. Coussios, Spatiotemporal evolution of cavitation dynamics exhibited by flowing microbubbles during ultrasound exposure, J. Acoust. Soc. Am. 132, 3538 (2012).

[10] C. D. Arvanitis, M. Bazan-Peregrino, B. Rifai, L. W. Seymour, and C. C. Coussios, Cavitation-enhanced extravasation for drug delivery, Ultrasound Med. Biol. 37, 1838 (2011).

[11] H. Arslan, I. Capar, G. Saygili, T. Gok, and M. Akcay, Effect of photon-initiated photoacoustic streaming on removal of apically placed dentinal debris, Int. Endod. J. 47, 1072 (2014).
[12] J. J. Kwan, R. Myers, C. M. Coviello, S. M. Graham, A. R. Shah, E. Stride, R. Carlisle, and C. C. Coussios, Ultrasound-propelled nanocups for drug delivery, Small 11, 5305 (2015).

[13] R. Myers, C. Coviello, P. Erbs, C. Rowe, J. Kwan, J. Foloppe, C. Crake, S. Finn, E. Jackson, J. M. Ballouls, C. Story, C. Coussios, and R. Carlisle, Polymeric cups for cavitation mediated delivery of oncolytic vaccinia virus, Mol. Ther. (to be published).

[14] R. M. Wagterveld, L. Boels, M. J. Mayer, and G. J. Witkamp, Visualization of acoustic cavitation effects on suspended calcite crystals, Ultrason. Sonochem. 18, 216 (2011).

[15] M. Arora, C. D. Ohl, and K. A. Morch, Cavitation Inception on Microparticles: A Self-Propelled Particle Accelerator, Phys. Rev. Lett. 92, 174501 (2004).

[16] J. R. T. Seddon, H. J. W. Zandvliet, and D. Lohse, Knudsen Gas Provides Nanobubble Stability, Phys. Rev. Lett. 107, 116101 (2011).

[17] S. Jones, G. Evans, and K. Galvin, Bubble nucleation from gas cavities-A review, Adv. Colloid Interface Sci. 80, 27 (1999).

[18] D. Lohse and X. Zhang, Surface nanobubbles and nanodroplets, Rev. Mod. Phys. 87, 981 (2015).

[19] T. G. Leighton, The Acoustic Bubble (Academic Press Limited, London, 1994), p. 312.

[20] B. M. Borkent, S. Gekle, A. Prosperetti, and D. Lohse, Nucleation threshold and deactivation mechanisms of nanoscopic cavitation, Phys. Fluids 21, 102003 (2009).

[21] J. J. Kwan, S. Graham, R. Myers, R. Carlisle, E. Stride, and C. C. Coussios, Ultrasound-induced inertial cavitation from gas-stabilizing nanoparticles, Phys. Rev. E 92, 023019 (2015).

[22] E. C. Gelderblom, H. J. Vos, F. Mastik, T. Faez, Y. Luan, T. J. A. Kokhuis, A. F. W. van der Steen, D. Lohse, N. de Jong, and M. Versluis, Brandaris 128 ultra-high-speed imaging facility: 10 years of operation, updates, and enhanced features, Rev. Sci. Instrum. 83, 103706 (2012).

[23] V. Garbin, D. Cojoc, E. Ferrari, E. Di Fabrizio, M. L. J. Overvelde, S. M. van der Meer, N. de Jong, D. Lohse, and M. Versluis, Changes in microbubble dynamics near a boundary revealed by combined optical micromanipulation and high-speed imaging, Appl. Phys. Lett. 90, 114103 (2007).

[24] B. L. Helfield, B. Y. C. Leung, and D. E. Goertz, The effect of boundary proximity on the response of individual ultrasound contrast agent microbubbles, Phys. Med. Biol. 59, 1721 (2014).

[25] See Supplemental Material at http://link.aps.org/ supplemental/10.1103/PhysRevApplied.6.044004 for a figure showing the representative acoustic responses from nanocups below and above the cavitation threshold and a figure showing their persistence with respect to the pulse number.

[26] H. G. Flynn, Generation of transient cavities in liquids by microsecond pulses of ultrasound, J. Acoust. Soc. Am. 72, 1926 (1982).

[27] B. M. Borkent, M. Arora, C. D. Ohl, N. De Jong, M. Versluis, D. Lohse, K. A. Morch, E. Klaseboer, and B. C. Khoo, The acceleration of solid particles subjected to cavitation nucleation, J. Fluid Mech. 610, 157 (2008). 
[28] C. D. Ohl and R. Ikink, Shock-Wave-Induced Jetting of Micron-Size Bubbles, Phys. Rev. Lett. 90, 214502 (2003).

[29] Y. Matsumoto and F. Takemura, Influence of internal phenomena on gas bubble motion. Effects of thermal diffusion, phase change on the gas-liquid interface and mass diffusion between vapor and noncondensable gas in the collapsing phase, JSME Int J. Ser. B 37, 288 (1994).
[30] E. J. Aymé-Bellegarda, Collapse and rebound of a gas-filled spherical bubble immersed in a diagnostic ultrasonic field, J. Acoust. Soc. Am. 88, 1054 (1990).

[31] W. Kreider, L. A. Crum, M. R. Bailey, and O. A. Sapozhnikov, Observations of the collapses and rebounds of millimeter-sized lithotripsy bubbles, J. Acoust. Soc. Am. 130, 3531 (2011). 